\title{
3D Scene Rendering Based on EEG Data Driven and the Application in Biofeedback Therapy
}

\author{
Xinliang Wei ${ }^{1,2}$ and Wei Sun ${ }^{1,2}$ \\ ${ }^{1}$ SUN Yat-sen University, Guangzhou, China \\ ${ }^{2}$ Key Laboratory of Information Technology (Ministry of Education), SUN Yat-sen University, Guangzhou, China
}

\begin{abstract}
Using human biological signs to drive the scene and control the object in the scene, has important significance in the development of the virtual reality technology. Through human biological signs to drive the virtual scene, the user get a better sense of immersion. They also can control their own situation through the feedback information of the scene, so that their physical condition can reach a good state. The technology is also important in the biofeedback therapy. This paper uses brain waves to control the opening of the scroll in the virtual scene, and to verify the feasibility of the biological signs to drive the virtual scene. What's more, it also analyze the application in biofeedback therapy briefly.
\end{abstract}

Keywords-EEG data driven; $3 D$ scene rendering; biofeedback therapy

\section{INTRODUCTION}

Virtual reality (VR) is an immersive interactive environment based on computable information. It takes the computer technology as the core, combined with other related science and technology (such as interactive technology, artificial intelligence, etc.), to generate a visual, auditory, tactile and other aspects of the environment with a high degree of approximation of the digital environment. Users can make use of other devices and interact with the environment, resulting in proximity to the feeling and experience of the corresponding real environment [1]. With the aid of input devices, including the keyboard, mouse, sensor data gloves, etc., users can interact with the virtual environment. Further, users take advantage of their own biological signs data (such as ECG, EEG, etc.) to drive the 3D scene. We take a special instrument to collect user's biological signs, and then the collected data are processed and analyzed, and applied to 3D scene.

The concept of biofeedback therapy is only beginning to be applied in medical science in recent years, and it is better than the traditional biological feedback method by using virtual reality technology to enhance the biofeedback treatment [2]. With 3D virtual reality technology, the user's physiological or pathological information is converted into a virtual reality scene. Users get feedback from the change of 3D scene, and adjust their physiological signs to control the visual scene changes, so that the scene trends to target state (physiological or pathological conditions achieve a good state). At last, user can control their special signs in order to obtain the effect of prevention and treatment.

In this paper, brain waves are used to drive a simple scenario which uses brain waves to control the opening and closing of scroll. The opening state of scroll reflects the user's brain wave state when user feel more nervous, the scroll opens slowly even closes while user feel more relaxed, the scroll open quickly. Users should control their relaxation degree to make scroll open entirely. What's more, Users can also view the corresponding brain data chart, and understand the specific brain wave number.

\section{3D SCENE RENDERING BASED ON EEG DATA}

\section{A. Data Collection}

The collection of brain wave need a special instrument, and this paper used MindWave Mobile as the collecting device. The EEG of cerebral cortex acquisition is microvolt signal, which main energy contained in $0.05 \sim 32 \mathrm{~Hz}$ band. It is weak signal which has low noise-signal ratio and is easy to receive the interference of the surrounding environment. Therefore, in order to enhance the effective components of the EEG signal, and suppress the noise, as well as filter and process the brain signal, this paper select MindWave Mobile acquisition instrument, which has advanced noise filtering technology, and resist a variety of interference in the environment in the daily life. Table 1 describes main parameters of MindWave Mobile.

TABLE I. Parameters of MindWaVe Mobile

\begin{tabular}{|l|l|}
\hline Parameter Type & Parameter Value \\
\hline Sampling Rate & $512 \mathrm{~Hz}$ \\
\hline Frequency Range & $3 \mathrm{~Hz}-100 \mathrm{~Hz}$ \\
\hline Common Mode Rejection Ratio & $\geq 105 \mathrm{db}$ \\
\hline Input Impedance & $\geq 10 \mathrm{M} \Omega$ \\
\hline Output Baud Rate & $1200,9600,57600$ \\
\hline Maximum Power Consumption & $15 \mathrm{~mA} @ 3.3 \mathrm{~V}$ \\
\hline Operating Voltage & $2.97-3.63 \mathrm{~V}$ \\
\hline
\end{tabular}




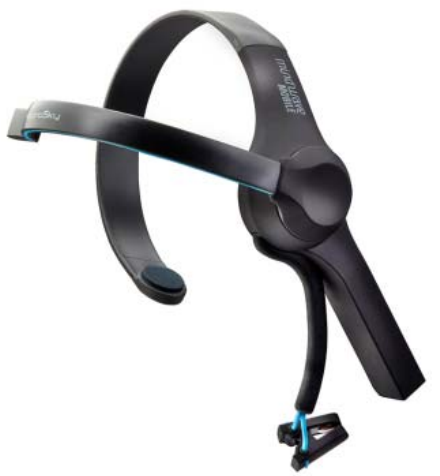

FIGURE I. PHOTO OF MINDWAVE MOBILE

\section{B. Data Analysis}

Our brain generates brain waves constantly, and in the electroencephalogram, we can see four kinds of brain waves as follow.

1) $\alpha$ wave $(8-13 \mathrm{~Hz})$ : When we feel relax and our brain are active, or when we are inspired, our brain will generate more $\alpha$ wave. The frequency of $\alpha$ wave is 8 to 13 times per second, and the average is 10 times. It is the basic rhythm of normal human brain waves, and if there is no external stimulus, its frequency is quite constant. When people stay in clear and quite space and close their eyes, the rhythm is most obvious. When people open their eyes or accept other stimuli, $\alpha$ wave will disappear immediately.

2) $\beta$ wave $(14-30 \mathrm{~Hz})$ : When we feel nervous or emotional, our brain will generate more $\beta$ wave. The frequency of $\beta$ wave is 14 to 30 times per second. We can divide $\beta$ wave into 3 kinds according to its degree: low $\beta$ wave $(12-15 \mathrm{~Hz}$, the rhythm of movement and we can also focus attention or coordination), midrange $\beta$ wave $(16-20 \mathrm{~Hz}$, thinking and we are aware of ourselves and surrounding environment), high $\beta$ wave $(21-30 \mathrm{~Hz}$, vigilant and excited).

3) $\theta$ wave (4-7Hz): When we feel sleepy, our brain will generate $\theta$ wave. The frequency of $\theta$ wave is 4 to 7 times per second. When adults feel frustrated or depressed, this kind of wave is most obvious. But $\theta$ wave is main ingredients of juvenile's EEG.

4) $\delta$ wave $(1-3 \mathrm{~Hz})$ : When we are in deep sleep, our brain will generate $\delta$ wave. The frequency of $\delta$ wave is 1 to 3 times per second. When we are baby or our intellectual development is not mature, our brain also generate this kind of wave.

MindWave Mobile acquisition instrument can directly obtain the original wave of various brain waves (16 bits). The data consists of two bytes, representing a single original wave sample while its range is from -32768 to 32767 , and its value is a 16 bits signed integer. The first byte of the value represents a high level, and the second byte represents a low level. If we rebuild the original value, we can simply move the first byte to the left of 8 bits, and press or the second byte as follow.

$$
\text { short raw }=(\text { Value }[0]<<8) \mid \text { Value[1]; }
$$

Value [0] is high byte, and Value[1] is low byte.

MindWave Mobile also provides a variety of current intensity of brain waves, which is a series of 3 byte unsigned integer output. For convenience, it also provides two parameters (attention degree and meditation degree) for developer to use. These two parameters reflect the brain's focus and relaxation state and this paper makes use of these two parameters to control the $3 \mathrm{D}$ scene.

\section{Scene Driven}

This paper made a simple demonstration program, using brain waves to drive the scroll in 3D scene to open or close. The flow of process is as following.

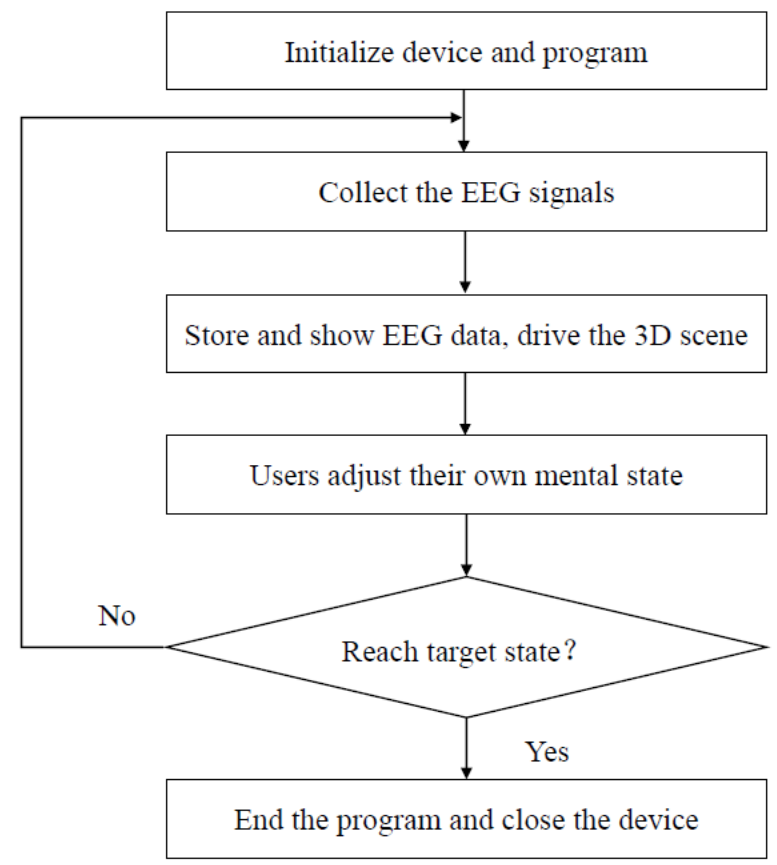

FIGURE II. THE FLOW OF PROCESS

Figure 2 describes the whole process of the demonstration:

a) Initialize the device and the program.

b) Collect the EEG signals at set intervals.

c) Store and show the EEG data, then use the data to control the $3 D$ scene.

d) Users adjust their own mental state according to the feedback of $3 D$ scene.

e) Judge the scene's state or users' state whether reach the target state or not.

f) If it doesn't reach the target state, repeat step 2, or end the program and close the device. 
Users will wear the MindWave Mobile to collect their brain wave signals. The program will store the signals data and show them into the mobile application. What's more, the program will provide the background music for users to enjoy. Some screenshots are as following.

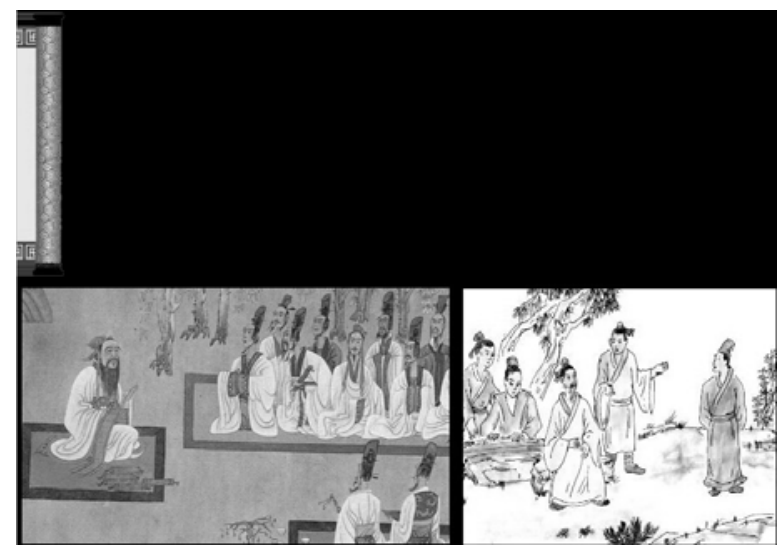

FIGURE III. SCROLL JUST OPEN

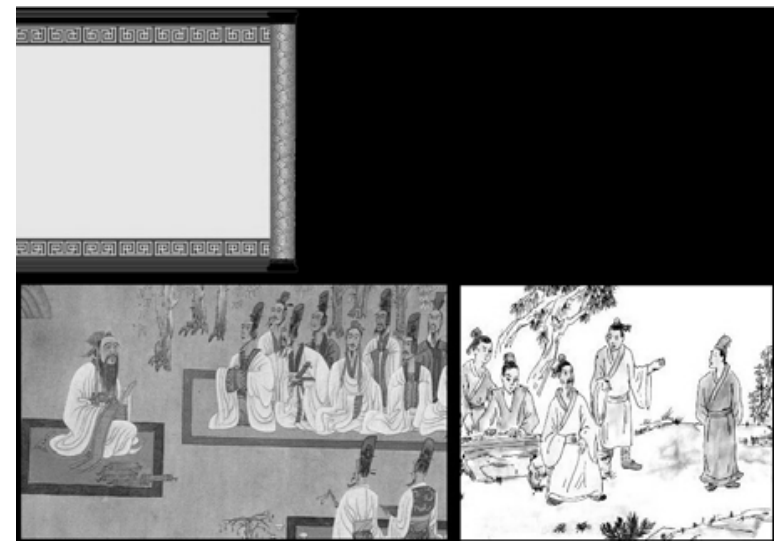

FIGURE IV. SCROLL IS OPENING

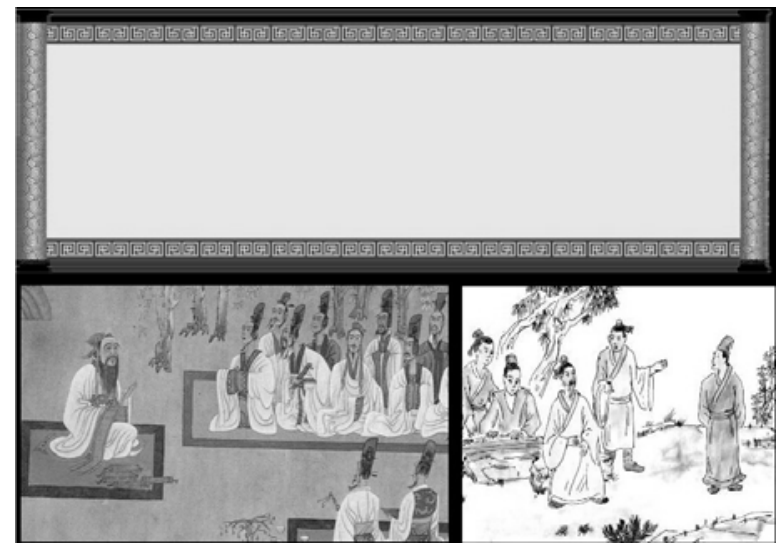

FIGURE V. SCROLL OPEN COMPLETELY

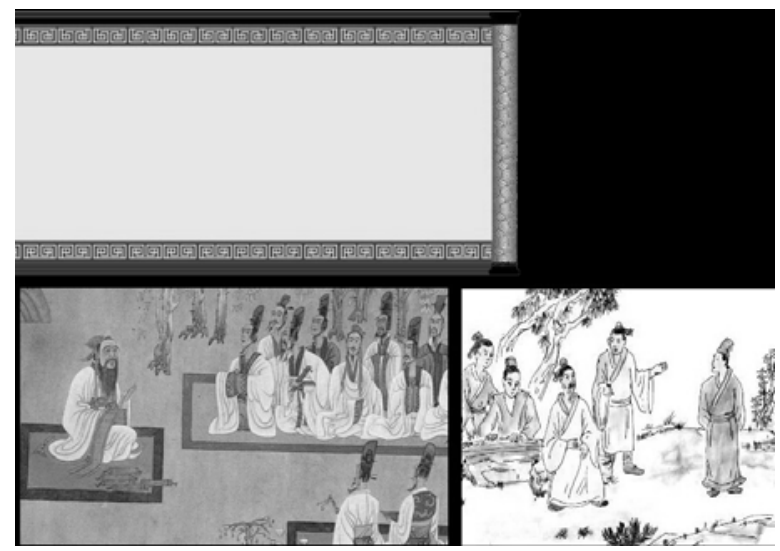

FIGURE VI. SCROLL IS CLOSING

The attention and meditation value is between 1 and 100 . If the value is between 40 and 60 , that means the attention and meditation is normal. If the value is between 60 and 80 , that means the attention and meditation is high. If the value is larger than 80 , the attention and meditation is very high and you focus on something or feel very relaxed. The screenshot of mobile app is figure VII.

\section{THE APPLICATION IN BIOFEEDBACK THERAPY}

Biological feedback technology is the focus of the research in the field of medicine. The theory basis is that the human being can control the body's characteristics through the interaction with the environment [3]. In this paper, the brain waves is the subject investigated. Traditional biofeedback therapy collect some physical signs through collecting device, and then transform signs data into acceptable form (chart, music, etc.), and give the feedback to users. Users can feel their own physical condition by a series of training and treatment, as well as the dynamic scene. Further, Users can control some physical signs and even treat specific ailments through their own adjustment and psychological suggestion.

The current biological feedback technology is to obtain the user's own signs information and make users adjust themselves. Virtual reality in this area is to strengthen the feedback information, increase the effect of information feedback. Compared to the traditional chart and music form, virtual reality can establish realistic feedback scenarios. Users in the course of treatment have a stronger sense of substitution, immersion and they can access to their own signs information changes as well as more intuitive feeling clearer. As the virtual reality is driven by the user's own signs data, there will be a unified and harmonious feeling rather than the bald instrument as well as the waveform during the treatment. Users can get a more powerful effect than the traditional way [4].

\section{CONCLUSIONS}

In this paper, the feasibility that using brain wave data to control the object in $3 \mathrm{D}$ scene is verified. Biological characteristics of the human body not only contain EEG and ECG, may also contain EMG, skin, skin temperature, EOG, blood pressure, blood volume, heart rate, and so on. All kinds of biological characteristics are likely to be the driving data in 
the virtual scene, and control the object in the $3 \mathrm{D}$ scene so that users can get a better sense of reality and immersion [5]. This paper also analyzes the effect of virtual reality technology in biological feedback treatment. Each organism may contain many kinds of diseases. The virtual scene can be more friendly to display the user's physiological or pathological characteristics, and can be more intuitive to tell the user feedback information, so that users make a corresponding adjustment, so as to achieve a good state and achieve the purpose of reducing or treating the disease.

In this paper, the current research is still relatively shallow, hoping to provide a reference for the future research of virtual reality technology in the field of biofeedback treatment. There are still many problems to be solved in the treatment of virtual reality and biofeedback therapy:

a) The real sense of the virtual scene is still limited to the screen $3 D$ effect, is not the true sense of the scene

b) Biofeedback therapy for different diseases or biological signs, need to develop a different virtual reality system, and the system reuse is low or there is no unified framework c) The cost of virtual equipment is very high, the treatment process between doctors and patients cannot reach the remote or mobile treatment

At present, only a part of problems that need to be solved are listed, and some problems in the future research are put forward and solved. Believe that the above problem is solved, virtual reality technology in the field of biological feedback treatment will bring better results.

\section{REFERENCES}

[1] Cho B H, Lee J M, Ku J H, et al. (2002) Attention enhancement system using virtual reality and EEG biofeedback, Proceedings of IEEE Virtual Reality, pp156-163

[2] Barbara B. Brown. (1977) Stress and the art of biofeedback. London, Harper and Row, pp50-78.

[3] Gary E. Schwartz, Jackson Beatty. (1977) Biofeedback: theory and research. New York, Academic Press, pp313-367.

[4] Obrist P A, Black A H, Brener J. (2007) Cardiovascular Psychophysiology: Current Issues in Response Mechanisms, Biofeedback and Methodology. Aldine De Gruyter.

[5] Barbara B. Brown. (1973) New mind, new body: bio-feedback: new directions for the mind. New York, Harper \& Row, pp213-342.

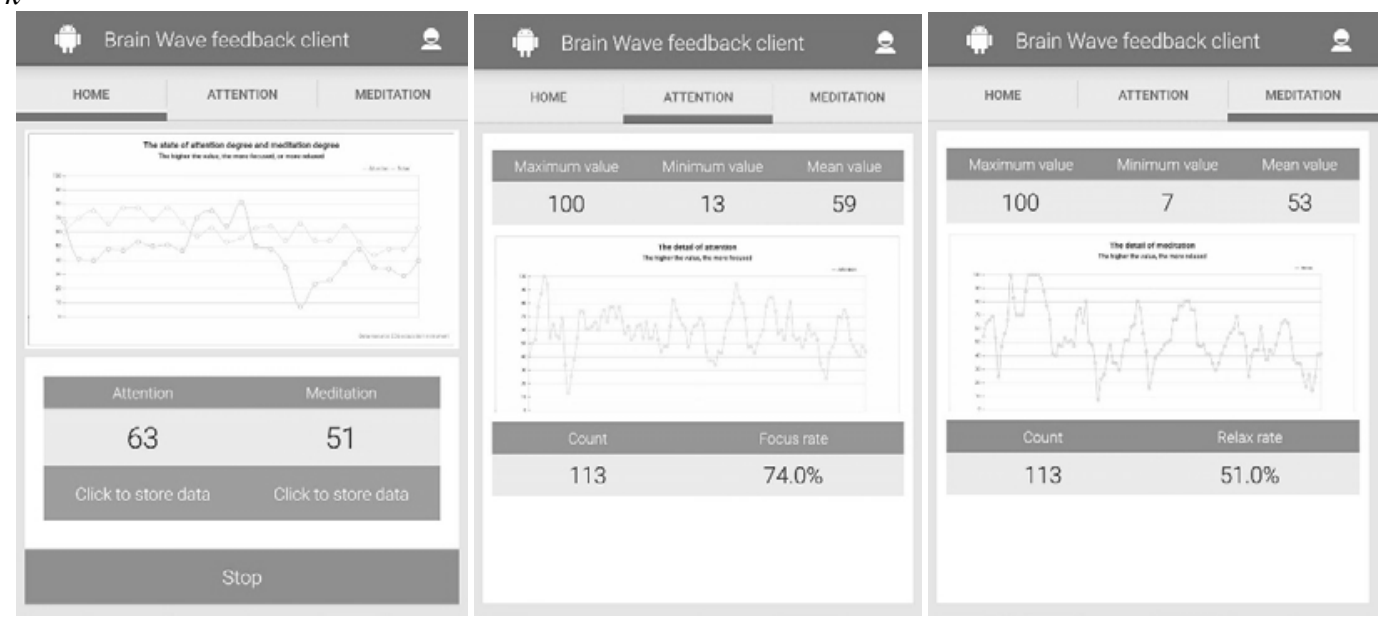

FIGURE VII. SCREENSHOT OF MOBILE APP ( TO SHOW THE VALUE) 